\title{
Fomento del trabajo autónomo en las prácticas de laboratorio de alumnos del grado de química: caso de estudio
}

\section{Promotion of self-employed work in the laboratory practices of students of the degree in chemistry: case study}

\author{
María Asunción López-Bascón ${ }^{1}$, Natividad Jurado-Campos², Encarnación \\ Romera-García ${ }^{3}$, Feliciano Priego-Capote ${ }^{4} \&$ Lourdes Arce $^{5}$
}

Fecha de recepción: 16/04/2019; Fecha de revisión: 08/06/2019; Fecha de aceptación: 03/09/2019

Cómo citar este artículo:

López-Bascón, M.A., Jurado-Campos, N., Romera-García, E., Priego-Capote, F. \& Arce, L. (2019). Fomento del trabajo autónomo en las prácticas de laboratorio de alumnos del grado de química: caso de estudio. Revista de Innovación y Buenas Prácticas Docentes, 8(3), 101-112.

Autor de Correspondencia: a92lobam@uco.es, nati jurado@hotmail.com, q02rogae@uco.es

\begin{abstract}
Resumen:
En este estudio se ha perseguido incrementar la participación de los alumnos en las prácticas de laboratorio. El motivo principal ha sido evitar que el alumno memorice los conceptos teóricos sin ser capaz de aplicarlos a la resolución de problemas reales, y así adquiera compentencias útiles para cuando se introduzca en el mundo laboral. La nueva metodología se desarrolló en una asignatura optativa del Grado de Química. Para abordar la nueva metodología propuesta en este proyecto, los alumnos elaboraron los protocolos de tres prácticas, sobre tres temas propuestos por el profesorado. Una vez supervisados dichos protocolos por el profesorado, los alumnos las realizaron de forma autónoma en el laboratorio. La evaluación de los alumnos se realizó mediante: elaboración de los protocolos de prácticas, realización de las prácticas de laboratorio (mediante rúbrica) y cuestionario sobre conceptos teóricos (mediante la herramienta Kahoot). Finalmente, se evaluó el grado de aceptación de la nueva metodología docente por parte del alumnado, para detectar aspectos a mejorar para el próximo año académico. Esta metodología ha permitido una mayor implicación del alumnado en las sesiones de laboratorio, ya que pudieron aplicar los conceptos teóricos adquiridos sobre Química Analítica y adquirir competencias, tales como capacidad de aprendizaje autónomo.

Palabras clave: competencias, evaluación por objetivos, trabajo autónomo, trabajo de laboratorio.
\end{abstract}

\begin{abstract}
:
In this study we have sought to increase the participation of students in laboratory practices. The main reason has been to prevent the student from memorizing the theoretical concepts without being able to apply them to the resolution of real problems, and thus acquire useful competences for when entering into the working world. The new methodology was developed in an optional subject of the Degree in Chemistry. Therefore, it was proposed that they themselves elaborate the protocols of practices, on subjects proposed by the teaching staff, and that they carried them out independently in the laboratory. The evaluation of the students' work was carried out through three activities: preparation of the practical protocols, carrying out of the laboratory practices (using a rubric) and questionnaire on theoretical concepts (using the Kahoot tool). Finally, the degree of acceptance of the new teaching methodology by the students was evaluated. This allowed to detect the aspects to improve for the next academic year. This methodology has allowed a greater involvement of the students in the laboratory sessions, since they could apply the theoretical concepts acquired on Analytical Chemistry and acquire competences, such as autonomous learning capacity. Key Words: competences, criterion-referenced evaluation, laboratory work, self-employed work.

\footnotetext{
${ }^{1}$ Universidad de Córdoba (España), a92lobam@uco.es; CÓDIGO ORCID: 0000-0002-0543-8357

2 Universidad de Córdoba (España), nati jurado@hotmail.com; CÓDIGO ORCID: 0000-0002-5761-9774

${ }^{3}$ Universidad de Córdoba (España), q02rogae@uco.es; CÓDIGO ORCID: 0000-0002-9048-0516

${ }^{4}$ Universidad de Córdoba (España), q72prcaf@uco.es; CÓDIGO ORCID: 0000-0003-0697-719X

${ }_{5}^{5}$ Universidad de Córdoba (España), lourdes.arce@uco.es; CÓDIGO ORCID: 0000-0002-7130-8446
} 


\section{INTRODUCCIÓN}

En los programas formativos antiguos (licenciaturas y diplomaturas), solo se tenía en cuenta la adquisición de conocimientos específicos por parte del alumnado. Un aspecto diferencial de la enseñanza de Grado, contemplada en el marco en el Espacio Europeo de Enseñanza Superior (EEES) y en el Marco Español de Cualificaciones (MECES), es la definición de programas formativos que implican conocimientos y competencias.

Para abordar estos cambios en el ámbito docente, tanto el profesorado como los alumnos se han enfrentado a una situación que principalmente se ha caracterizado por la participación más activa por parte del alumnado en el proceso de aprendizaje. Por su parte, el profesorado se ha visto obligado a adaptar su metodología a esta nueva tendencia. En este contexto, la innovación juega un papel muy importante para estimular a los alumnos a la participación más activa en la docencia, durante el proceso de aprendizaje.

En el Grado de Química se contempla como competencia básica la "Capacidad de aprendizaje autónomo para el desarrollo continuo profesional" (Santamaría Antonio, 2004, p. 81). Dicha competencia se encuentra recogida en el Acuerdo de la Comisión Andaluza del Título de Grado en Química (Ministerio de Educación y Ciencia, 2007, p. 2). La importancia de esta competencia resulta evidente si tenemos en cuenta que los graduados universitarios deben estar preparados para su interacción en un mundo profesional cada vez más cambiante. Por tanto, la universidad debe prestar a sus alumnos las herramientas y estrategias necesarias para que éstos puedan aprender de manera autónoma y continuada durante toda su vida; de ahí surge el término "life long learning" (Knapper \& Cropley, 2000, p. 2). Los principales problemas se plantean a la hora de buscar metodologías para que los alumnos adquieran dicha competencia, y a su vez, para evaluar la adquisición de esta.

Actualmente, las prácticas de laboratorio que se imparten en las asignaturas de los grados de ciencias no son motivadoras para el alumnado. En la mayoría de ellas los alumnos solo tienen que seguir un guion que el profesor les entrega al inicio de esta y deben reproducirlo. Con esta metodología docente el alumnado no suele implicarse y los conceptos no se asimilan correctamente. Por ello, creemos que la mejor forma de que el alumnado se motive es que se le planteen problemas reales que podrán encontrar en su vida profesional y que tendrán que resolver mediante la aplicación de los conceptos teóricos. Con dicha actividad los alumnos no solo podrán adquirir la competencia en aprendizaje autónomo, sino que también mejorarán su capacidad de síntesis y de planificación, búsqueda de información de interés, toma de decisiones, razonamiento crítico, trabajo en equipo y resolución de problemas.

La evaluación fundada en competencias es un aspecto esencial en el paradigma docente en el que se desarrolla el espacio europeo de enseñanza superior (Sánchez Garrido \& Córdoba Medina, 2010, p. 10). La rúbrica es una herramienta importante para evaluar cualquier actividad debido a su flexibilidad. Esto la convierte en una herramienta útil en la evaluación por competencias, ya que éstas se pueden desglosar en actividades más concretas para su evaluación (Cano, 2015, p. 266). Un complemento a la rúbrica es la evaluación basada en conceptos mediante herramientas interactivas como por ejemplo Kahoot! (Brand, 2013). Esta herramienta además de evaluar los conceptos adquiridos genera un ambiente distendido de aprendizaje, ya que los alumnos son muy receptivos al uso de nuevas tecnologías en clase.

La nueva metodología desarrollada se aplicó a la asignatura optativa Química Analítica Aplicada que se imparte en el segundo cuatrimestre del tercer curso del Grado de Química (Arce-Jimenez, 2018, p. 1). Esta asignatura tiene como objetivo aplicar los conocimientos teóricos y prácticos, adquiridos en las asignaturas previas ofertadas por 
el Departamento de Química Analítica de la Universidad de Córdoba, al análisis de muestras reales. En concreto con esta asignatura se persigue adquirir los conocimientos necesarios para abordar correctamente la toma y el tratamiento de muestra, así como la selección de las técnicas de separación y determinación más adecuadas en análisis clínico, de alimentos, ambiental y toxicológico.

El objetivo general del presente estudio es introducir el concepto de trabajo autónomo por parte del alumnado en las sesiones prácticas de laboratorio en la asignatura de Química Analítica Aplicada del Grado de Química. Los objetivos específicos derivados del objetivo general han sido: (1) dar las herramientas necesarias al alumnado para mejorar su trabajo autónomo en la resolución de problemas en el ámbito químico; (2) permitir al alumnado un aprendizaje activo y reflexivo, que supere la pura transmisión de conocimientos teóricos; (3) evaluar dicha metodología a través de la evaluación del alumnado en competencias y conceptos.

\section{DESARROLLO DE LA EXPERIENCIA DE INNOVACIÓN}

El estudio se desarrolló durante las sesiones prácticas de aula y de laboratorio de la asignatura optativa Química Analítica Aplicada. El número reducido de alumnos/as que estaban matriculados en la asignatura, un total de 16, facilitó la evaluación por competencias y por conceptos. El alumnado se dividió en seis grupos de trabajo (cuatro grupos de tres alumnos/as y dos grupos de dos alumnos/as).

Cada sesión de prácticas de laboratorio tuvo como objetivo la resolución de uno de los problemas planteados por parte del profesor previamente en el aula (prácticas de aula). Por este motivo se planificaron tres sesiones de prácticas de laboratorio, para que todos los alumnos pudiesen trabajar cada problema planteado. Dichas sesiones tuvieron una duración de seis horas/sesión y se llevaron a cabo en el laboratorio de Practicas del Departamento de Química Analítica los días 29 de marzo, 5 de abril y 26 de abril de 2019. A continuación, se detallan las experiencias realizadas en orden cronológico:

- En la primera sesión de prácticas de aula (fecha de realización 4 de marzo,) se informó al alumnado sobre la nueva metodología que se iba a implantar, la evaluación y la planificación de trabajo que se iba a llevar a cabo. Finalmente, se le plantearon tres problemas reales de diferentes ámbitos profesionales (agroalimentario y ambiental). Los problemas planteados fueron los siguientes:

- Problema 1: Determinación de alcaloides (cafeína, teofilina y teobromina) en refrescos (de cola, con extracto de té y bebidas energéticas) mediante cromatografía de líquidos acoplada a detector ultravioleta (HPLC-UV).

- Problema 2: Determinación de éster metílicos de ácidos grasos (FAMEs) en aceites vegetales (oliva virgen extra, girasol refinado y maíz) mediante cromatografía de gases acoplada a detector de ionización por llama (GC-FID).

- Problema 3: Determinación de oxidantes totales en el aire atmosférico del entorno del Campus de Rabanales.

- Durante la segunda sesión de prácticas de aula (fecha de realización 15 de marzo), se enseñó al alumnado a utilizar todas las herramientas disponibles para la búsqueda bibliográfica de información, que les pudiesen ser útil para la resolución de los 
problemas planteados. Los alumnos tuvieron acceso a este material a través del espacio Moodle de la asignatura (GQUIM-3-100473-1-1) durante todo el curso.

- Con estas herramientas el alumnado (organizado en grupos) dispuso de 3 semanas para buscar y proponer metodologías que permitiesen la resolución de los problemas planteados. Durante este tiempo estuvieron en continuo contacto con el profesorado, mediante sesiones de tutoría, para la resolución de las dudas que les iban surgiendo. Las sesiones de tutoría fueron tanto presenciales como mediante correo electrónico, según la disponibilidad del alumnado.

- Cinco días antes de la realización de las sesiones de prácticas de laboratorio, cada grupo envió al profesorado el protocolo definitivo que iban a seguir durante las sesiones de laboratorio. Puesto que todo el alumnado hizo un buen uso de las sesiones de tutoría, el $80 \%$ de los protocolos entregados eran válidos para resolver los problemas planteados. El profesor devolvió al alumnado los protocolos con correcciones y sugerencias de mejora.

- Se puso a disposición del alumnado todo el material básico y específico de laboratorio necesario (material de vidrio, pipetas automáticas, estándares comerciales, muestras reales del problema, etc.). Además, durante toda la sesión se les permitió usar el resto del material disponible en el laboratorio del departamento, bajo la supervisión de los profesores. En la Figura 1 se muestra una fotografía de un puesto de trabajo.

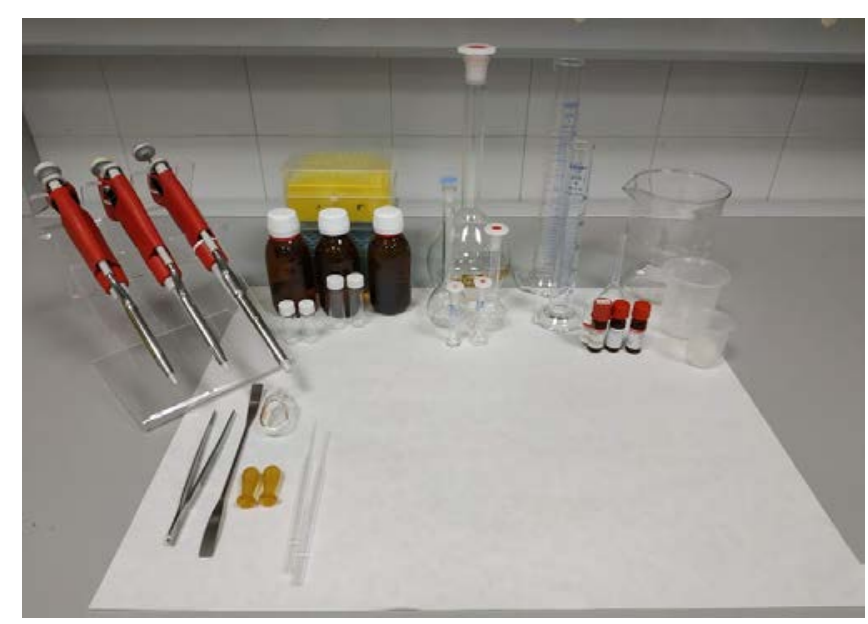

Figura 1. Ejemplo de uno de los puestos de trabajo utilizados durante las sesiones de prácticas de laboratorio. Fuente: Elaboración propia.

El profesorado utilizó la rúbrica (Figura 2) para evaluar el trabajo desarrollado por el alumnado durante dichas sesiones. Por otra parte, el desarrollo cronológico durante la sesión de prácticas de laboratorio fue como sigue:

- Durante los primeros 45 min de cada sesión de prácticas se explicó la técnica analítica (LC-UV, GC-FID ó fotometría) que se iba a utilizar, dispositivos o herramientas necesarias, y además, se contextualizó el problema analítico planteado. También se informó al alumno del material de laboratorio del que disponía, gestión de residuos y equipamiento de protección individual que se les podía proporcionar.

- Durante la siguiente hora los grupos explicaron al profesorado y a los demás compañeros/as el protocolo que ellos proponían para resolver el problema. De este modo, se expusieron todas las metodologías y se llevó a cabo un debate 
crítico con el alumnado, con el fin de decidir cuál era la más adecuada. La selección del mejor método se evaluó desde el punto de vista analítico, económico y medioambiental.

- Durante el resto de la sesión (4 h) se llevó a cabo la resolución del problema con la metodología acordada. El objetivo principal de esta estrategia docente fue iniciar al alumnado en el trabajo autónomo de laboratorio con una visión crítica.

- Tras la finalización de las sesiones de prácticas de laboratorio se le permitió al alumnado mejorar el protocolo que habían elaborado. Se estableció el día 13 de mayo de 2019 como fecha límite para la entrega de dicho protocolo.

- Para finalizar el estudio, el día 21 de mayo de 2019 se llevó a cabo un breve cuestionario para evaluar los conceptos adquiridos durante las sesiones de prácticas haciendo uso de la herramienta interactiva Kahoot!. Al finalizar el cuestionario se recogió la opinión del alumnado sobre esta nueva metodología a través de un cuestionario anónimo.

El material utilizado durante toda la experiencia se detalla en el siguiente apartado.

\subsection{Materiales y métodos utilizados}

En el Moodle de la asignatura (GQUIM-3-100473-1-1) se puso a disposición del alumnado el siguiente material: (i) información sobre los problemas que debían resolver; (ii) plan de trabajo que se iba a realizar; y (iii) las herramientas para la búsqueda de información necesaria para la resolución de los problemas. Dichas herramientas se explicaron durante los seminarios con ejemplos concretos, como se ha comentado anteriormente.

La evaluación del alumnado se llevó a cabo mediante tres herramientas:

1. Los alumnos entregaron el protocolo realizado durante las sesiones de prácticas. Con él se evaluó la capacidad de presentar de forma clara y concisa y ante un público de su misma área la forma en que finalmente decidieron resolver el problema analítico planteado por el profesorado. De manera, que cualquier persona pudiera usar dicho protocolo para reproducir el método propuesto por los alumnos.

2. La rúbrica se utilizó para la evaluación del trabajo llevado a cabo en el laboratorio por los alumnos. El objetivo de esta fue aplicar una evaluación por competencias. Se evaluó la competencia objeto de estudio "Capacidad de aprendizaje autónomo para el desarrollo continuo profesional", y otras de interés para el Grado de Química. En la tabla 1 se muestra la rúbrica utilizada.

3. La herramienta interactiva Kahoot! se utilizó para la evaluación por conceptos. De este modo se comprobó que los alumnos habían adquirido los conceptos estudiados durante las sesiones de prácticas de laboratorio. Se desarrolló un cuestionario con 15 preguntas relacionadas con las tres sesiones de prácticas de laboratorio que tuvieron los alumnos. 


\begin{tabular}{|c|c|c|c|c|}
\hline & Deficiente (0) & Regular (1) & Correcto (2) & Destacable (3) \\
\hline Atención & $\begin{array}{l}\text { Está distraído } \\
\text { durante todas } \\
\text { las } \\
\text { explicaciones e } \\
\text { indicaciones del } \\
\text { profesor }\end{array}$ & $\begin{array}{l}\text { Con facilidad se } \\
\text { distrae en las } \\
\text { explicaciones y } \\
\text { realización de } \\
\text { tareas }\end{array}$ & $\begin{array}{l}\text { Está atento a las } \\
\text { explicaciones en } \\
\text { todo momento }\end{array}$ & $\begin{array}{l}\text { Está atento a las } \\
\text { explicaciones en } \\
\text { todo momento y } \\
\text { toma notas de las } \\
\text { mismas }\end{array}$ \\
\hline Ritmo de trabajo & $\begin{array}{l}\text { No trabaja } \\
\text { durante la } \\
\text { práctica }\end{array}$ & $\begin{array}{l}\text { Trabaja de } \\
\text { forma } \\
\text { discontinua y es } \\
\text { necesario } \\
\text { indicarle que } \\
\text { debe hacer }\end{array}$ & $\begin{array}{l}\text { Trabaja de forma } \\
\text { constante }\end{array}$ & $\begin{array}{l}\text { Trabaja de forma } \\
\text { constante y } \\
\text { optimiza el tiempo }\end{array}$ \\
\hline Trabajo en equipo & $\begin{array}{l}\text { No trabaja en } \\
\text { equipo }\end{array}$ & $\begin{array}{l}\text { Tiene dificultad } \\
\text { para trabajar en } \\
\text { equipo }\end{array}$ & $\begin{array}{l}\text { Trabaja en } \\
\text { equipo, pero tiene } \\
\text { dificultades para } \\
\text { tener en cuenta } \\
\text { las opiniones de } \\
\text { sus compañeros }\end{array}$ & $\begin{array}{l}\text { Trabaja en equipo, } \\
\text { ayuda a sus } \\
\text { compañeros y tiene } \\
\text { en cuenta la } \\
\text { opinión de todos } \\
\text { sus compañeros }\end{array}$ \\
\hline Destreza & $\begin{array}{l}\text { No tiene manejo } \\
\text { del material de } \\
\text { laboratorio }\end{array}$ & $\begin{array}{l}\text { Dificultad en el } \\
\text { manejo del } \\
\text { material de } \\
\text { laboratorio }\end{array}$ & $\begin{array}{l}\text { Tiene } \\
\text { conocimiento del } \\
\text { manejo del } \\
\text { material del } \\
\text { laboratorio }\end{array}$ & $\begin{array}{l}\text { Tiene conocimiento } \\
\text { del manejo del } \\
\text { material del } \\
\text { laboratorio y es } \\
\text { capaz de resolver } \\
\text { los problemas que } \\
\text { surgen de manera } \\
\text { autónoma }\end{array}$ \\
\hline $\begin{array}{l}\text { Preparación de las } \\
\text { disoluciones y reactivos }\end{array}$ & $\begin{array}{l}\text { Desconoce } \\
\text { cómo preparar } \\
\text { las disoluciones } \\
\text { y reactivos. }\end{array}$ & $\begin{array}{l}\text { Prepara con } \\
\text { dificultad las } \\
\text { disoluciones y } \\
\text { reactivos, y } \\
\text { necesita ayuda } \\
\text { en todo } \\
\text { momento. }\end{array}$ & $\begin{array}{l}\text { Prepara } \\
\text { correctamente las } \\
\text { disoluciones y } \\
\text { reactivos, pero } \\
\text { necesita ayuda } \\
\text { para tener en } \\
\text { cuenta el volumen } \\
\text { necesario, la } \\
\text { concentración, } \\
\text { etc. }\end{array}$ & $\begin{array}{l}\text { Prepara } \\
\text { correctamente las } \\
\text { disoluciones y } \\
\text { reactivos, teniendo } \\
\text { en cuenta el } \\
\text { volumen necesario, } \\
\text { concentración, etc. }\end{array}$ \\
\hline $\begin{array}{l}\text { Conocimientos teóricos y } \\
\text { aplicación en el } \\
\text { laboratorio. Ejemplo: } \\
\text { conoce el fundamento de } \\
\text { las técnicas utilizadas, } \\
\text { conoce las ecuaciones } \\
\text { necesarias para preparar } \\
\text { disoluciones, etc. }\end{array}$ & $\begin{array}{l}\text { No tiene } \\
\text { conocimiento de } \\
\text { la teoría }\end{array}$ & $\begin{array}{l}\text { Tiene dudas } \\
\text { sobre la teoría y } \\
\text { necesita ayuda } \\
\text { del profesor } \\
\text { para aplicarla en } \\
\text { el laboratorio. }\end{array}$ & $\begin{array}{l}\text { Tiene } \\
\text { conocimiento de } \\
\text { la teoría, pero } \\
\text { necesita ayuda } \\
\text { del profesor para } \\
\text { aplicarla en el } \\
\text { laboratorio }\end{array}$ & $\begin{array}{l}\text { Tiene conocimiento } \\
\text { de la teoría y la } \\
\text { aplica en el } \\
\text { laboratorio. }\end{array}$ \\
\hline $\begin{array}{l}\text { Obtención de resultados } \\
\text { (cuantificación, curva de } \\
\text { calibrado, etc.) }\end{array}$ & $\begin{array}{l}\text { No sabe cómo } \\
\text { llevar a cabo } \\
\text { una } \\
\text { cuantificación }\end{array}$ & $\begin{array}{l}\text { Tiene dudas } \\
\text { sobre cómo } \\
\text { llevar a cabo la } \\
\text { cuantificación }\end{array}$ & $\begin{array}{l}\text { Sabe llevar a } \\
\text { cabo una } \\
\text { cuantificación, } \\
\text { pero tiene dudas } \\
\text { sobre el } \\
\text { procedimiento }\end{array}$ & $\begin{array}{l}\text { Sabe llevar a cabo } \\
\text { una cuantificación y } \\
\text { lo realiza } \\
\text { correctamente }\end{array}$ \\
\hline
\end{tabular}

Figura 2. Rúbrica utilizada para la evaluación por competencias del alumnado.

Fuente: Elaboración propia. 


\begin{tabular}{|c|c|c|c|c|}
\hline & Deficiente (0) & Regular (1) & Correcto (2) & Destacable (3) \\
\hline $\begin{array}{l}\text { Razonamiento crítico y } \\
\text { discusión de resultados }\end{array}$ & $\begin{array}{l}\text { No discute los } \\
\text { resultados ni } \\
\text { obtiene } \\
\text { conclusiones }\end{array}$ & $\begin{array}{l}\text { Tiene dificultad } \\
\text { para discutir los } \\
\text { resultados y } \\
\text { obtener una } \\
\text { conclusión. } \\
\text { Necesita ayuda } \\
\text { del profesor. }\end{array}$ & $\begin{array}{l}\text { Discute los } \\
\text { resultados y } \\
\text { obtiene } \\
\text { conclusiones } \\
\text { correctas, pero le } \\
\text { cuesta realizar un } \\
\text { razonamiento } \\
\text { crítico y proponer } \\
\text { mejoras }\end{array}$ & $\begin{array}{l}\text { Es capaz de } \\
\text { realizar un } \\
\text { razonamiento } \\
\text { crítico sobre los } \\
\text { resultados } \\
\text { obtenidos, } \\
\text { proponiendo } \\
\text { mejoras }\end{array}$ \\
\hline $\begin{array}{l}\text { Seguridad en el } \\
\text { laboratorio }\end{array}$ & $\begin{array}{l}\text { No utiliza la } \\
\text { bata, guantes y } \\
\text { no lleva el pelo } \\
\text { recogido. } \\
\text { Desconoce las } \\
\text { normas de } \\
\text { seguridad }\end{array}$ & $\begin{array}{l}\text { Utiliza la bata, } \\
\text { guantes y lleva } \\
\text { el pelo recogido. } \\
\text { Desconoce las } \\
\text { normas de } \\
\text { seguridad. }\end{array}$ & $\begin{array}{l}\text { Utiliza la bata, } \\
\text { guantes y lleva el } \\
\text { pelo recogido. } \\
\text { Conoce las } \\
\text { normas de } \\
\text { seguridad, pero } \\
\text { tiene dificultad } \\
\text { para aplicarlas. }\end{array}$ & $\begin{array}{l}\text { En todo momento } \\
\text { utiliza la bata, } \\
\text { guantes y lleva el } \\
\text { pelo recogido. } \\
\text { Conoce las normas } \\
\text { de seguridad y las } \\
\text { aplica } \\
\text { correctamente } \\
\text { (utiliza la campana, } \\
\text { no mezcla residuos } \\
\text { peligrosos, etc.) }\end{array}$ \\
\hline Gestión de residuos & $\begin{array}{l}\text { No se preocupa } \\
\text { por la gestión de } \\
\text { los residuos ni } \\
\text { conoce como se } \\
\text { lleva a cabo }\end{array}$ & $\begin{array}{l}\text { Solo en } \\
\text { ocasiones se } \\
\text { preocupa por la } \\
\text { gestión de los } \\
\text { residuos y la } \\
\text { lleva a cabo }\end{array}$ & $\begin{array}{l}\text { Se preocupa por } \\
\text { la gestión de los } \\
\text { residuos y la lleva } \\
\text { a cabo con ayuda } \\
\text { del profesor }\end{array}$ & $\begin{array}{l}\text { Se preocupa por la } \\
\text { gestión de los } \\
\text { residuos y la lleva a } \\
\text { cabo sin ayuda del } \\
\text { profesor (conoce } \\
\text { los procedimientos) }\end{array}$ \\
\hline
\end{tabular}

Figura 2. Rúbrica utilizada para la evaluación por competencias del alumnado (continuación).

Fuente: Elaboración propia.

En la tabla 1 se refleja el porcentaje asignado a cada una de las actividades evaluadas. En total, la calificación de las sesiones de prácticas de aula y laboratorio suponían un $20 \%$ de la calificación total de la asignatura.

Tabla 1.

Evaluación de las sesiones de prácticas de aula y laboratorio.

\begin{tabular}{lc}
\hline Actividad desarrollada & $\begin{array}{c}\text { Porcentaje de la } \\
\text { calificación (\%) }\end{array}$ \\
\hline 1. Protocolo elaborado por el alumnado & 10 \\
\hline 2. Trabajo en sesiones de laboratorio (rúbrica) & 5 \\
\hline 3. Cuestionario de conceptos teóricos relacionados con las prácticas & 5 \\
de laboratorio (Kahoot!) & \\
\hline Fuente: Elaboración propia. &
\end{tabular}


La evaluación de la nueva metodología por parte de los alumnos se hizo a través de un cuestionario anónimo, donde los alumnos pudieron dar su opinión acerca de la nueva metodología, la organización por parte del profesorado, los sistemas de evaluación, etc. En la tabla 2 se muestra dicho cuestionario. Consideramos una parte imprescindible la evaluación y mejora continua de todas las metodologías educativas para adaptarnos al alumnado y a sus nuevas necesidades. Por ello, se propuso dicho cuestionario donde el alumnado tuvo la opción de expresar libremente los aspectos positivos y negativos de esta nueva metodología. Con esto se pretendía que no fuese un cuestionario con preguntas cerradas, sino que ellos pudiesen expresarse.

Tabla 2.

Cuestionario anónimo para recoger la opinión del alumnado acerca de la nueva metodología aplicada.

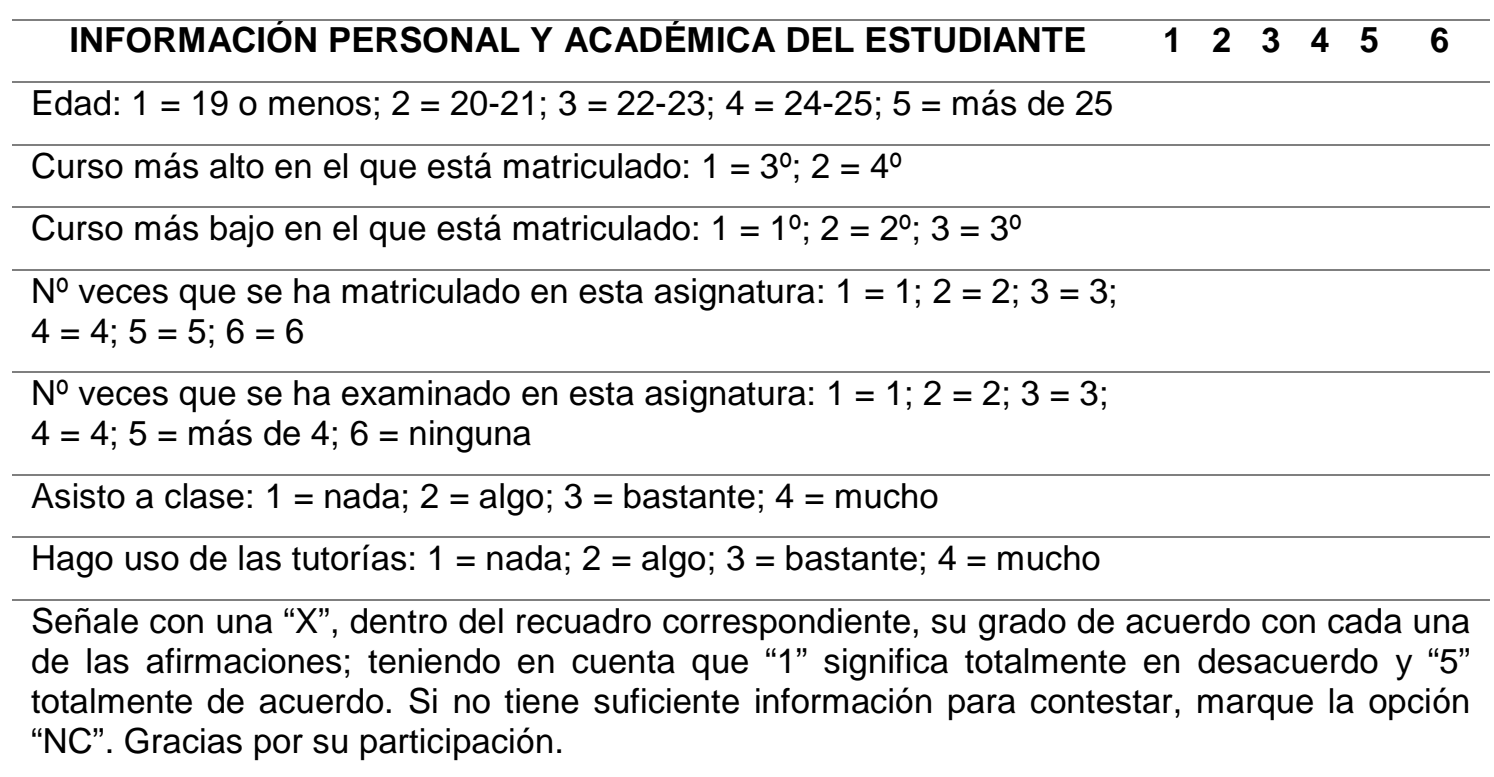

\section{DESARROLLO DE LA DOCENCIA}

$\begin{array}{llllll}1 & 2 & 3 & 4 & 5 & \text { NC }\end{array}$

1. Los problemas reales planteados son atractivos y de interés

2. La información inicial aportada para la resolución de los problemas es suficiente

3. El tiempo dedicado en los seminarios para desarrollar las sesiones de prácticas de laboratorio es suficiente

4. Las herramientas de búsqueda facilitadas en los seminarios han resultado de ayuda para la resolución de los problemas

5. Tenía clara la información que debía incluir en los guiones de prácticas

6. El tiempo del que se ha dispuesto para la entrega de los guiones de prácticas es suficiente

7. El tiempo invertido en la preparación de los guiones de prácticas se adecua a las horas de trabajo no presenciales correspondientes a esta asignatura

Fuente: Elaboración propia. 
Tabla 2.

Cuestionario anónimo para recoger la opinión del alumnado acerca de la nueva metodología aplicada (continuación).

8. Las profesoras han estado disponibles para la resolución de cualquier duda acerca de la elaboración de los guiones de prácticas

9. Las correcciones y recomendaciones hechas por las profesoras me han permitido disponer de una metodología analítica antes de la realización de la práctica

10. El tiempo del que se ha dispuesto en el laboratorio para la realización de la práctica es suficiente

11. En el laboratorio disponía de todo el material necesario para la realización de la práctica

12. EL profesorado se interesa por el grado de comprensión de sus explicaciones

13. El profesorado motiva al alumno durante la sesión de prácticas de laboratorio

14. Las preguntas del cuestionario realizado en la plataforma Kahoot! habían sido resueltas durante las sesiones prácticas

\section{RESULTADOS}

$\begin{array}{llllll}1 & 2 & 3 & 4 & 5 & \text { NC }\end{array}$

15. La práctica me ha permitido comprender mejor los conceptos teóricos aprendidos en esta y otras asignaturas

16. Las actividades desarrolladas han contribuido a que mejore las siguientes destrezas:

\subsection{Resolución de problemas}

16.2 Razonamiento crítico

16.3 Capacidad de aprendizaje autónomo para el desarrollo continuo profesional

16.4 Capacidad para organizar, dirigir y ejecutar tareas que se realicen en un laboratorio químico

17. En general, la nueva metodología docente es más beneficiosa, para el alumno, que la forma tradicional de abordar las prácticas de laboratorio

Fuente: Elaboración propia.

\section{RESULTADOS}

Los resultados obtenidos derivan de la evaluación del alumnado con esta nueva metodología, y de la evaluación de la nueva metodología por parte del alumnado. Con el cuestionario anónimo realizado, pudimos conocer alguna información personal y académica relevante acerca del grupo. El rango de edad estaba comprendido entre 2023 años, y ninguno de ellos se había examinado previamente de dicha asignatura. Todo el grupo asiste a clase de forma habitual, aunque el $40 \%$ reconoce que nunca o solo a veces, hace uso de las tutorías para resolver dudas. El 100\% del alumnado es la primera 
vez que se ha matriculado de la asignatura. Al tratarse de una asignatura optativa se puede deducir que el alumnado que se matricula tiene interés por el contenido que se trata en ella. Por este motivo, se decidió elegir una asignatura optativa para la implantación de la nueva metodología.

\subsection{Evaluación del alumnado con la nueva metodología aplicada}

La evaluación del alumnado con esta nueva metodología se llevó a cabo como se describe en el apartado 4. En la tabla 3 se muestran las calificaciones obtenidas en cada una de las actividades evaluadas por el profesorado. El $93.75 \%$ del alumnado obtuvo una calificación superior a 8.5 (sobre 10) en la calificación final de las sesiones prácticas de la asignatura ( $20 \%$ de la calificación global).

En los resultados de las diferentes actividades de forma individual hubo una calificación de 0 para el cuestionario de conceptos teóricos (Kahoot!). Sin embargo, esta calificación está justificada, ya que dicho alumno/a no asistió a la clase donde se realizó el cuestionario y no justificó la falta de asistencia.

En cuanto a los resultados globales de los alumnos, la actividad Kahoot! es en la que peores resultados han obtenido (un 8 sobre 10 de media) y donde los alumnos tuvieron resultados más dispares entre sí, a la vista de la los valores de desviación relativa. Esto podría significar que algunos alumnos no han adquirido individualmente los conocimientos teóricos de las prácticas llevadas a cabo en su totalidad, aunque hayan sabido plantear bien su elaboración (evaluación mediante el protocolo) y la hayan ejecutado de forma correcta en el laboratorio (evaluación mediante la rúbrica).

Tabla 3.

Calificaciones medias y desviación estándar obtenida en cada actividad por el alumnado. Se muestra la calificación máxima y mínima obtenida en cada actividad.

\begin{tabular}{ccccc}
\hline Calificaciones/Actividad & $\begin{array}{c}\text { Trabajo en sesiones } \\
\text { de laboratorio } \\
\text { (rúbrica, 5\%) }\end{array}$ & $\begin{array}{c}\text { Protocolo } \\
\text { elaborado por } \\
\text { el alumando } \\
\mathbf{( 1 0 \% )}\end{array}$ & $\begin{array}{c}\text { Cuestionario } \\
\text { conceptos } \\
\text { teóricos } \\
\text { (Kahoot!, 5\%) }\end{array}$ & $\begin{array}{c}\text { Calificación } \\
\text { final (20\%) }\end{array}$ \\
\hline Media $\pm \mathrm{SD}^{*}$ & $4.26 \pm 0.18$ & $9.5 \pm 0.33$ & $4.04 \pm 1.13$ & $17.8 \pm 1.14$ \\
\hline Calificación máxima & 4.56 & 10.00 & 5.00 & 19.44 \\
\hline Calificación mínima & 4.00 & 9.08 & 0.00 & 14.02 \\
\hline
\end{tabular}

*Desviación estándar relativa.

Fuente: Elaboración propia.

\subsection{Evaluación de la nueva metodología aplicada}

Como se ha comentado anteriormente, es clave conocer la opinión del alumnado acerca de las nuevas metodologías que se aplican en el aula. Esto permite una mejora continua de las mismas, ya que en ocasiones nuestra percepción como profesorado es muy distinta a la percepción del alumnado.

En general, los resultados obtenidos en estas encuestas han sido muy positivos y enriquecedores para el profesorado. El 100\% del alumnado reconoce la importancia de esta nueva metodología para su desarrollo profesional y la valoran de forma muy positiva.

Por otro lado, hay cosas que se pueden mejorar para el próximo curso y que el alumnado ha dejado recogido en sus cuestionarios: (i) el horario, la realización en el 
mismo día de seis horas de prácticas de laboratorio es agotador, ya que deben estar mentalmente muy activos durante el desarrollo de toda la sesión; (ii) la dificultad a la hora de encontrar información útil para la resolución de los problemas y la realización de los protocolos; y por último, (iii) el tiempo para la búsqueda de información y desarrollo de los protocolos lo consideran insuficiente. Este último aspecto también ha sido registrado mediante el bloque del cuestionario "Desarrollo de la Docencia", donde el $40 \%$ del alumnado considera que el tiempo dedicado en los seminarios para desarrollar las sesiones de prácticas de laboratorio ha sido insuficiente. El profesorado tendrá en cuenta estos resultados para mejorar en los siguientes cursos académicos.

En la tabla 4 se muestra un extracto de los resultados obtenidos en el cuestionario anónimo realizado al alumnado (tabla 3), concretamente de la sección "Resultados". Cabe destacar que el 81.3\% de los alumnos están totalmente de acuerdo en el apartado "15. La práctica me ha permitido comprender mejor los conceptos teóricos aprendidos en esta y otras asignaturas". Del mismo modo, 96.75\% de los alumnos están de acuerdo o totalmente de acuerdo con la afirmación "17. En general, la nueva metodología docente es más beneficiosa, para el alumno, que la forma tradicional de abordar las prácticas de laboratorio". Estos resultados son alentadores, ya que el alumnado valora la metodología y la consideran importante, del mismo modo que el profesorado.

Tabla 4.

Resultados obtenidos para la evaluación de la nueva metodología docente aplicada.

\begin{tabular}{|c|c|c|c|c|c|c|}
\hline RESULTADOS & 1 & 2 & 3 & 4 & 5 & NC \\
\hline $\begin{array}{l}\text { 15. La práctica me ha permitido comprender mejor los conceptos teóricos } \\
\text { aprendidos en esta y otras asignaturas }\end{array}$ & & & 1 & 2 & 13 & \\
\hline \multicolumn{7}{|l|}{$\begin{array}{l}\text { 16. Las actividades desarrolladas han contribuido a que mejore las } \\
\text { siguientes destrezas: }\end{array}$} \\
\hline 16.1 Resolución de problemas & & & 1 & 6 & 9 & \\
\hline 16.2 Razonamiento crítico & & & 1 & 8 & 7 & \\
\hline $\begin{array}{l}\text { 16.3 Capacidad de aprendizaje autónomo para el desarrollo continuo } \\
\text { profesional }\end{array}$ & & & & 7 & 9 & \\
\hline $\begin{array}{l}\text { 16.4 Capacidad para organizar, dirigir y ejecutar tareas que se realicen } \\
\text { en un laboratorio químico }\end{array}$ & & & 1 & 8 & 7 & \\
\hline $\begin{array}{l}\text { 17. En general, la nueva metodología docente es más beneficiosa, para } \\
\text { el alumno, que la forma tradicional de abordar las prácticas de laboratorio }\end{array}$ & & & 1 & 4 & 11 & \\
\hline
\end{tabular}

Fuente: Elaboración propia.

\section{CONCLUSIONES}

La experiencia ha demostrado la importancia de fomentar el trabajo autónomo en el alumnado del Grado de Química para su desarrollo personal y profesional, tal y como lo recogen las competencias básicas de dicho grado. El alumnado finaliza el Grado de 
Química con muchos conocimientos teóricos, pero en la mayoría de los casos son incapaces de aplicarlos a problemas reales que se les plantean.

Esta nueva metodología ha permitido una mayor implicación del alumnado en las sesiones prácticas de laboratorio, en las que lo convencional es realizar un ejercicio práctico siguiendo un protocolo establecido por el profesor. Además, mediante esta metodología el alumnado pudo aplicar los conceptos teóricos adquiridos en las asignaturas de Química Analítica de forma autónoma, además de adquirir competencias como: (i) capacidad de resolución de problemas, (ii) razonamiento crítico, (iii) capacidad de aprendizaje autónomo, y (iv) capacidad para organizar, dirigir y ejecutar tareas de laboratorio, (v) diseño de las metodologías de trabajo necesarias. El propio alumnado reconoce la adquisición de dichas competencias en el cuestionario anónimo realizado al final de la asignatura.

Consideramos que, en el contexto de la educación superior, esta metodología puede ser aplicada en los diferentes Grados de Ciencias. Se recomienda su utilización en cursos avanzados, en los que los alumnos dispongan de conocimientos suficientes para la resolución de problemas complejos.

\section{AGRADECIMIENTOS}

Los miembros agradecen a la Universidad de Córdoba la financiación recibida en el proyecto de Innovación Docente 2018-2-2002. N. Jurado-Campos, M.A. López-Bascón y E. Romera-García están agradecidas al Ministerio de Ciencia por la beca FPU (FPU15/0639, FPU15/02373, FPU15/03704, respectivamente). que les ha permitido participar en la docencia realizada en dicho trabajo.

\section{REFERENCIAS}

Arce-Jiménez, L. (2018). Guía docente de la asignatura Química Analítica Aplicada impartida en la Universidad de Córdoba curso 2018-2019. Recuperado de: http://bit.ly/2MGoa15

Brand, J. (2013). Kahoot! Consultada el 21 de mayo de 2019 desde https://kahoot.com/

Cano, E. (2015). The rubrics as an assessment tool of competency in higher education: use or abuse?. Revista de curriculum y formación del profesorado. 19, 265-280. Recuperado de: http://bit.ly/2M3vQvd

Knapper, C. Cropley, AJ. (2000). Lifelong learning in higher education (3 ed.). London, Psychology Press.

Ministerio de Educación y Ciencia (2007). Real Decreto 1393/2007 de 29 de octubre, por el que se establece la ordenación de las enseñanzas universitarias oficiales. Boletín Oficial del Estado (BOE). Recuperado de: http://bit.ly/2IFuO6e

Sánchez-Garrido, D. \& Córdoba-Medina, E. (2010). Manual docente para la autoformación en competencias básicas (1 ed.) Antequera, Consejería de Educación, Junta de Andalucía. Recuperado de: http://bit.ly/2nCAmr2

Santamaría, J. (2004). Libro Blanco del Título de Grado en Química, Madrid, Agencia Nacional de Evaluación de la Calidad y Acreditación (ANECA). Recuperado de: http://bit.ly/2Vta8nn

Universidad de Córdoba (2018). Competencias Grado Química. Recuperado de: http://bit.ly/320L0a4 